\title{
CONCOMITANT INFECTION OF LEPTOSPIROSIS WITH DENGUE AT A TERTIARY CARE HOSPITAL IN CHENNAI
}

\author{
B. Ananthi ${ }^{1}, \mathrm{G}$. Sumathi ${ }^{2}$
}

\section{HOW TO CITE THIS ARTICLE:}

B. Ananthi, G. Sumathi. "Concomitant Infection of Leptospirosis with Dengue at a Tertiary Care Hospital in Chennai". Journal of Evolution of Medical and Dental Sciences 2014; Vol. 3, Issue 08, February 24;

Page: 1823-1827, DOI: $10.14260 /$ jemds/2014/2063

ABSTRACT: BACKGROUND: Concomitant infection of leptospirosis with dengue has been reported in places where these diseases are endemic and leptospirosis may be overlooked in such cases. Recognition of leptospirosis is especially important since antimicrobial agents can reduce its severity and duration. Failure to identify leptospirosis in these patients will result in high mortality rate for this infection. Hence this study was conducted to have a detailed knowledge and awareness about the concomitant infection of leptospirosis with dengue. MATERIALS AND METHODS: Patients admitted to the medical ward of Government General Hospital, Chennai with fever of $\geq 5$ days and of the age group twelve years and above were evaluated for leptospirosis by doing the screening test MSAT (Macroscopic Slide Agglutination Test). All patients who tested positive for MSAT were further confirmed by MAT (Microscopic Agglutination Test). Those patients who tested positive for leptospirosis were investigated for concomitant infection with dengue utilising IgM ELISA. Patients positive for both leptospirosis and dengue were taken up for the study and were evaluated for relevant clinical features and laboratory profile. RESULTS: 143 patients were found to be positive for leptospirosis and among them 4 (2.8\%) patients were positive for the concomitant dengue infection. In the four concomitant infection patients, two were males and two were females, all in the age group of 20 to 30 years. Fever and myalgia were the predominant symptoms in these patients. Thrombocytopenia was seen in 3 (75\%) patients. With fluid resuscitation, platelet transfusion, appropriate drug and supportive therapy all the four patients recovered. No mortality was observed in this study. CONCLUSION: Increased awareness of concomitant infection and an enhanced ability to diagnose it early in illness is necessary so that appropriate treatment and supportive measures can be given to reduce the morbidity and mortality. Leptospiral illness may be a significant component in patients with concomitant infections and hence it is essential to evaluate for leptospirosis in all patients with fever for leptospirosis, especially in endemic areas.

KEYWORDS: Leptospirosis, Dengue, Concomitant infections.

INTRODUCTION: Leptospirosis and dengue are two major urban health problems associated with high mortality ${ }^{1,2}$. Leptospirosis is a zoonotic infection caused by the pathogenic spirochaete from the genus Leptospira and dengue is a mosquito borne arboviral infection caused by any one of the four serotypes of dengue virus. Higher incidence of leptospirosis and dengue occur during monsoon and post-monsoon periods (July to January). Both the diseases have similar clinical manifestation during the initial phase and cause difficulties in clinical diagnosis. Both the diseases have well-recognized severe forms which can be fatal ${ }^{3}$. Due to simultaneous transmission during rainy season, it is expected that concomitant infections may occur ${ }^{4}$ and management of concomitant infections is complicated by their similar clinical presentations. As outbreak of dengue is common and for longer periods as compared to leptospirosis, the diagnosis of later is often overshadowed 5 . Leptospirosis is 
difficult to diagnose in remote areas since diagnostic tools are usually unavailable ${ }^{6}$ and moreover in a dengue outbreak, if an acutely febrile patient is found to be dengue-positive, dengue is assumed to be the sole cause of the fever. Failure to recognize leptospirosis in such concomitant infection patients means a delay in the initiation of its proper therapy and possible consequences of serious complications ${ }^{7}$. Hence increased awareness and detailed knowledge about the concomitant infection of leptospirosis with dengue is necessary, so that a high index of suspicion is maintained and diagnosed early for initiating appropriate management and for better outcome.

MATERIALS AND METHODS: This study was done in Leptospirosis Research Cell, Institute of Microbiology, Madras Medical College, Chennai and the study was reviewed and approved by the Institutional Ethical Committee. Patients admitted to the medical wards of Government General Hospital, Chennai with fever due to infectious disease of duration five days and more were investigated for leptospirosis. Patients who tested positive for leptospirosis by MSAT (Macroscopic Slide Agglutination Test) were investigated for concomitant infection with dengue utilizing IgM ELISA. All patients tested positive by MSAT were further confirmed by MAT (titers of 1:80). Patients positive for both leptospirosis and dengue were taken up for the study and evaluated for relevant clinical features and laboratory profile. Patients aged 12 years and above were taken up for the study and the period of study was for one year. Only patients with fever due to infectious disease were taken and those with fever associated with malignancies, autoimmune diseases were excluded from the study.

RESULTS: 143 patients were found to be positive for leptospirosis by MSAT and among them 4 (2.8\%) patients were positive for the concomitant dengue infection. All the four patients were positive for dengue IgM antibodies. Out of the 4 patients, 2 were males and 2 were females, all in the

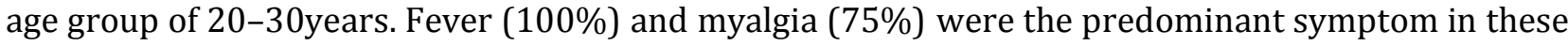
patients. Splenomegaly in one patient (25\%), hepatomegaly in one patient (25\%) and hemorrhage in one patient (25\%) were the signs observed (Table 1). The characteristic dengue rash was absent in all these patients. The differential count in dengue dual infection patients showed increased polymorphs percentage in 2 patients (50\%) and increased lymphocytes percentage in 2 patients (50\%). Platelet count was normal in one patient (25\%) and decreased in 3 patients $(75 \%)$. Out of these three cases with decreased platelets, one had platelet count less than 50, 000 (Table 2), which indicates poor prognosis. Liver parameters and renal parameters were normal in all the four concomitant infection patients. With fluid resuscitation, platelet transfusion, appropriate drug and supportive therapy all the four concomitant infection patients recovered. No mortality was observed in this study.

DISCUSSION: Leptospirosis and dengue are two infectious diseases of global importance and are two of the many medical conditions responsible for undifferentiated febrile illness, especially in tropical and subtropical regions ${ }^{1}$. The clinical manifestations of leptospirosis and dengue range from mild self-limited febrile illness to a severe and potentially fatal illness characterized by jaundice, renal failure, thrombocytopenia and hemorrhage. The emergence of dual infection has further complicated the situation. Both typically occur during the rainy season ${ }^{8}$ and rapid laboratory confirmation of the infecting pathogen is generally not available. Several studies have shown that leptospirosis is often 
confused with dengue and remains underdiagnosed in endemic regions ${ }^{9}$. An enhanced ability to distinguish leptospirosis from dengue and the ability to pick up dual infection in endemic population would guide clinicians and public health personnel to initiate appropriate therapy and reduce mortality ${ }^{3}$.

In our study 143 patients were found to be positive for leptospirosis by MSAT. MSAT is a simple sensitive test used to diagnose current leptospirosis and hence this test was used as a screening test. In single samples, positive MSAT confirms current leptospiral infection. ${ }^{10}$ It is preferable to do MAT in samples which are positive by MSAT, as during epidemics, large numbers of samples have to be tested and it is not possible to do MAT alone as it is a complicated test.

In the present study concomitant infection with dengue was seen in 4 cases $(2.8 \%)$ as compared to $8 \%$ in Levett P. N. et al study in Barbados. ${ }^{11}$ All the four patients were positive for dengue IgM antibodies. The characteristic dengue rash was absent in all these patients which is contrary to Levett $\mathrm{P} N$ et al study in Barbados in which all cases had dengue rash ${ }^{11}$. Thrombocytopenia which is the hallmark of dengue infection was seen in $75 \%$ of patients in our study.

Deranged hepatorenal function is very common in both dengue and leptospirosis and indicates bad prognosis ${ }^{3}$. But in our study liver parameters and renal parameters were normal in all the four concomitant infection patients. This indicates that all the four patients were in the early stage of diseases. We diagnosed them early, because of the readily available diagnostic facilities in our tertiary health care hospital which are not there in remote settings. Appropriate drug and supportive therapy were given to all the four patients. All of them recovered and no mortality was observed in this study.

During the second half of 1995, an epidemic of dengue type I infection occurred. In that period, leptospirosis mortality was twice the average, suggesting that some cases of leptospirosis were being misdiagnosed and treated inappropriately. ${ }^{11}$ During a dengue epidemic, when public health information is widely broadcast, patients will usually associate a febrile illness with aches and pains with dengue. It is important to educate both patients and physicians that leptospirosis occurs in the same setting, and patients should be carefully assessed and monitored. Due to high prevalence of both the diseases in our region, incidence of concomitant infections are increasing and needs to be recognized early to initiate appropriate treatment and reduce mortality. Recognition of leptospirosis in such concomitant infection patients is extremely important as antibiotic therapy in leptospirosis leads to favorable outcome, while dengue as such has no specific treatment and is treated symptomatically.

CONCLUSION: Concomitant infection of leptospirosis with dengue occurs in geographical areas endemic to both infections. Identifying and treating such concomitant infections are essential for rapid recovery and to prevent complications. Hence current knowledge and increased awareness about the concomitant infections are necessary so that a high index of suspicion will be maintained to diagnose them early. Leptospirosis being a condition having definitive antibiotic therapy, should always be evaluated in all patients with fever particularly in endemic regions as early initiation of antibiotic therapy can reduce morbidity and mortality significantly. 


\begin{tabular}{|l|c|}
\hline \multicolumn{1}{|c|}{ Signs and symptoms } & No. of cases (\%) \\
\hline Fever & $4(100 \%)$ \\
\hline Myalgia & $3(75 \%)$ \\
\hline Bleeding manifestations & $1(25 \%)$ \\
\hline Hepatomegaly & $1(25 \%)$ \\
\hline Splenomegaly & $1(25 \%)$ \\
\hline Rash & - \\
\hline Conjunctival suffusion & - \\
\hline Abdominal pain & - \\
\hline Jaundice & - \\
\hline \multicolumn{2}{|c|}{ Table 1: Clinical signs and symptoms } \\
in dual infection patients (n=4)
\end{tabular}

\begin{tabular}{|c|c|}
\hline Parameters & No. of cases $(\%)$ \\
\hline \multicolumn{2}{|l|}{ 1. Differential count } \\
\hline Polymorphs raised & $2(50 \%)$ \\
\hline Lymphocytes raised & $2(50 \%)$ \\
\hline \multicolumn{2}{|l|}{ 2. $\quad$ Platelet count/cu.mm } \\
\hline$>2.5$ lakhs & $1(25 \%)$ \\
\hline 1.0 -1.5 lakhs & $2(50 \%)$ \\
\hline$<50,000$ & $1(25 \%)$ \\
\hline 3. Elevated ESR & $4(100 \%)$ \\
\hline
\end{tabular}

Table 2: Laboratory parameters in dual infection patients $(n=4)$

\section{REFERENCES:}

1. Meguins LC, Junior HODM. Leptospirosis and dengue co-infection in a Brazilian Amazon patient. Rev Pan-Amaz Saude, 2010; 1(4): 97-99.

2. Sharp TM, Bracero J, Rivera A, Shieh WJ, Bhatnagar J, Rivera-Diez I, Hunsperger E, MunozJordan J, Zaki SR, Tomashek KM. Fatal Human Co-infection with Leptospira spp. and Dengue Virus. Emerg Infect Dis, 2012; 18(3): 878-880.

3. Kumar A, Balachandran V, Dominic A, Dinesh KR, Karim S, Rao G. Serological evidence of leptospirosis and dengue coinfection in an endemic region in South India. Ann Trop Med Public Health, 2012; 5: 286-290.

4. Bal AM. Unusual clinical manifestations of leptospirosis. J Postgrad Med, 2005; 51: 179-183.

5. Mishra B, Singhal L, Sethi S Ratho RK. Leptospirosis Coexistent with Dengue Fever: A Diagnostic Dilemma. J Glob Infect Dis, 2013; 5(3): 121-122.

6. Loganathan N, Ramalingam S, Ravishankar D, Shivakumar S. Co-Infection of Malaria and Leptospirosis - A Hospital Based Study from South India. Nat J Res Com Med, 2012; 1(2): 117119.

7. Wongsrichanalai C, Murray CK, Gray M, Miller RS, Mcdaniel P, Liao WJ, Pickard AL, Magill AJ. CoInfection with malaria and leptospirosis. Am. J. Trop. Med. Hyg, 2003; 68(5): 583-585. 


\section{ORIGINAL ARTICLE}

8. Zaki SA, Shanbag P. Clinical manifestations of dengue and leptospirosis in children in Mumbai: an observational study. Infection, 2010; 38(4): 285-91.

9. Ellis T, Imrie A, Katz AR, Effler PV. Under recognition of Leptospirosis during a dengue fever outbreak in Hawaii. Vector borne and zoonotic diseases, 2008; 8(4): 541-547.

10. Shivakumar S, Sumathi G, Krishnakumar B. Clinical profile of leptospirosis in North ChennaiDiagnosis utilizing Modified Faine's criteria. (Paper presented in APICON 2007, GOA).

11. Levett PN, Branch SL Edwards CN. Detection of dengue infection in patients investigated for leptospirosis in Barbados. Am J Trop Med Hyg, 2000; 62(1): 112-114.

\section{AUTHORS:}

1. B. Ananthi

2. G. Sumathi

\section{PARTICULARS OF CONTRIBUTORS:}

1. Assistant Professor, Department of Microbiology, ACS Medical College \& Hospital, Chennai, Tamilnadu, India.

2. Former Director, Institute of Microbiology, Madras Medical College \& Government Hospital, Chennai, Tamilnadu, India.

\section{NAME ADDRESS EMAIL ID OF THE} CORRESPONDING AUTHOR:

Dr. B. Ananthi, Assistant Professor, ACS Medical College \& Hospital, Velappan Chavadi, Chennai - 600077, Tamilnadu, India. E-mail: ananthiram1166@gmail.com

Date of Submission: 03/02/2014.

Date of Peer Review: 04/02/2014.

Date of Acceptance: 10/02/2014.

Date of Publishing: 18/02/2014. 